(REVIEW ARTICLE)

\title{
The road to becoming a certified robotic surgeon
}

\author{
Alejandro Diaz Giron Gidi 1, *, Regina Faes-Petersen ${ }^{2}$, Fabiola Nuccio Giordano ${ }^{3}$ and Eduardo \\ Villegas Tovar ${ }^{1}$
}

${ }^{1}$ General, Gastrointestinal and Robotic Surgeon, Department of Surgery Hospital Médica Sur México.

${ }^{2}$ General Surgery Fellow, Hospital Regional de Alta Especialidad de la Península de Yucatán, México.

3 Department of Anesthesiology, Hospital Médica Sur México.

Publication history: Received on 11 July 2020; revised on 17 July 2020; accepted on 18 July 2020

Article DOI: https://doi.org/10.30574/wjarr.2020.7.1.0253

\begin{abstract}
Surgical educators are challenged with introducing new technologies into general surgery training. There has been a rapid and widespread adoption of the robotic surgical system with a lag in the development of a comprehensive training and credentialing framework. A literature search on robotic surgical training techniques and benchmarks were conducted to provide an evidence-based road map for the development of a robotic surgical skills for the novice robotic surgeon. A structured training curriculum is suggested incorporating evidence-based training techniques and benchmarks for progress. This usually involves sequential progression from observation, case assisting, acquisition of basic robotic skills in the dry and wet lab setting along with achievement of individual and team-based non-technical skills, modular console training under supervision, and finally independent practice. There is a need for a standardized curriculum for training and assessment of robotic surgeons to proficiency, followed by high stakes testing for certification. A standardized process for certifying the skills of a robotic surgeon has begun to emerge.
\end{abstract}

Keywords: Surgical Procedures; Minimally Invasive; Robotic surgery; Continuing medical education

\section{Introduction}

Achieving surgical competence is a complex process that involves the attainment of knowledge, judgment, professionalism, and surgical skill. [1].

Robotic surgery has improved minimally invasive surgery, shortening the learning curve, and conferring increased dexterity for surgeons. The platform is very different from other forms of surgery, and if training is not comprehensive and well structured, patients can be put at risk [2, 3].

Since its approval by the US Food and Drug Administration (FDA) in 2000, the use of robot assisted laparoscopic surgery has surpassed that of pure laparoscopy for not only radical prostatectomy but also dismembered pyeloplasty and partial nephrectomy [4, 5]. Between 2007 and 2011, the annual number of total robotics cases according to Intuitive Surgical increased by nearly $400 \%$ in the United States (Fig. 1) [6].

\section{Robotic Platforms Currently Available for Practice}

Currently, there are several Food and Drug Administration (FDA) approved systems that are available to practicing surgeons and surgical trainees. A brief overview of these systems and future platforms is provided to lay a foundation for the complex task of creating and incorporating a universal robotics-training program into General Surgery education [7].

\footnotetext{
* Corresponding author: Alejandro Diaz Giron Gidi
} 


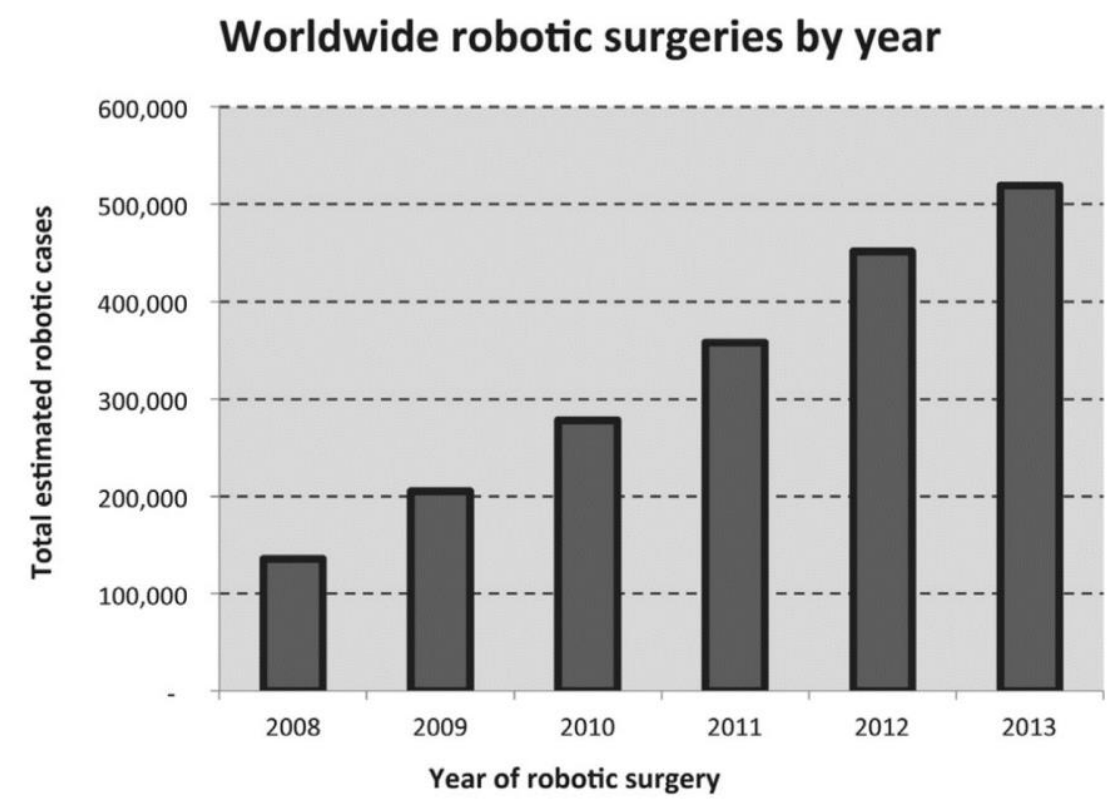

Figure 1. Estimated number of robot-assisted cases worldwide. (Data from Intuitive Surgical investor presentation, 2014. Available at http://investor.intuitivesurgical.com/phoenix.zhtml?c5122359\&p5irol-irhome.

\section{Da Vinci: Intuitive Surgical}

FDA approved since 2000, Intuitive Surgical's da Vinci robot gained popularity as the first system to house both surgical instruments and advanced imaging for general laparoscopic surgery. The most recent iterations of the da Vinci system are the da Vinci SP which is a single port operating platform and the da Vinci Ion which is a bronchoscope. The most commonly used platform is the da Vinci XI (Fig. 2) which consists of a closed master console with two cameras that generate a 3D/HD view of the operative site, a patient cart with four robotic arms with wristed instruments, and a vision cart. In addition, the high-definition 3D optics, tremor filtration, motion scaling and a comfortable user interface optimize the precision of the system [8]. Finally, the presence of Firefly near-infrared technology provides image-guided identification of key landmarks such as blood vessels and biliary structures using tissue fluorescence [9].
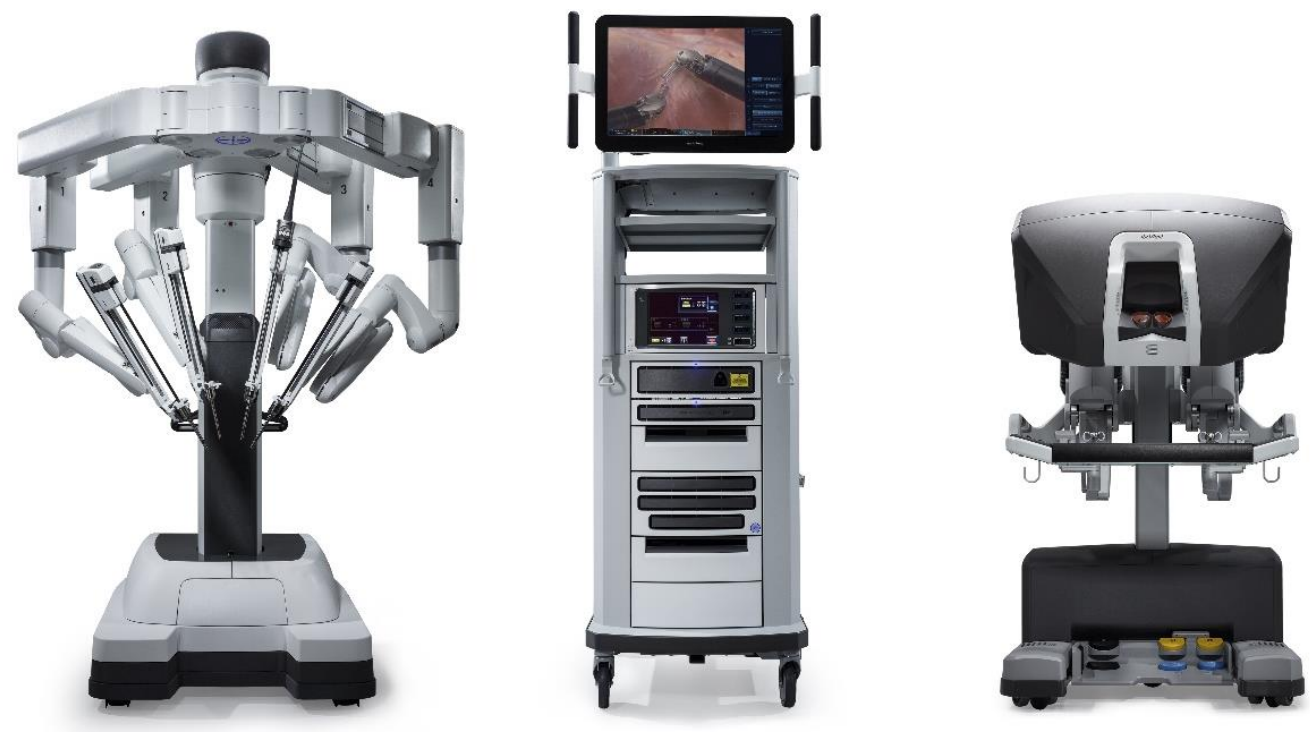

Figure 2 Da Vinci Image, Xi, Trio of System Components. Image of complete da Vinci Xi System including the patient side cart, vision cart and the surgeon console. 


\section{Requirements for Robotics Certification}

In 2007, the Society of American Gastrointestinal and Endoscopic Surgeons (SAGES) and the Minimally Invasive Robotic Association (MIRA) published a consensus statement putting forth guidelines for the educational requirements and certification for robotic surgery. With the broader adaptation of robotic surgery from pelvic specialties such as urology and gynecology into general surgery, it was critical to confirm that the training to support an expanded role of robotic surgery ensured patient safety [10].

\section{Certificate of da Vinci ${ }^{\circledR}$ System Training}

The Certificate of da Vinci System Training is not a replacement for any hospital's credentialing requirements and is not a representation of clinical competence, as Intuitive only trains on the use of the da Vinci system.

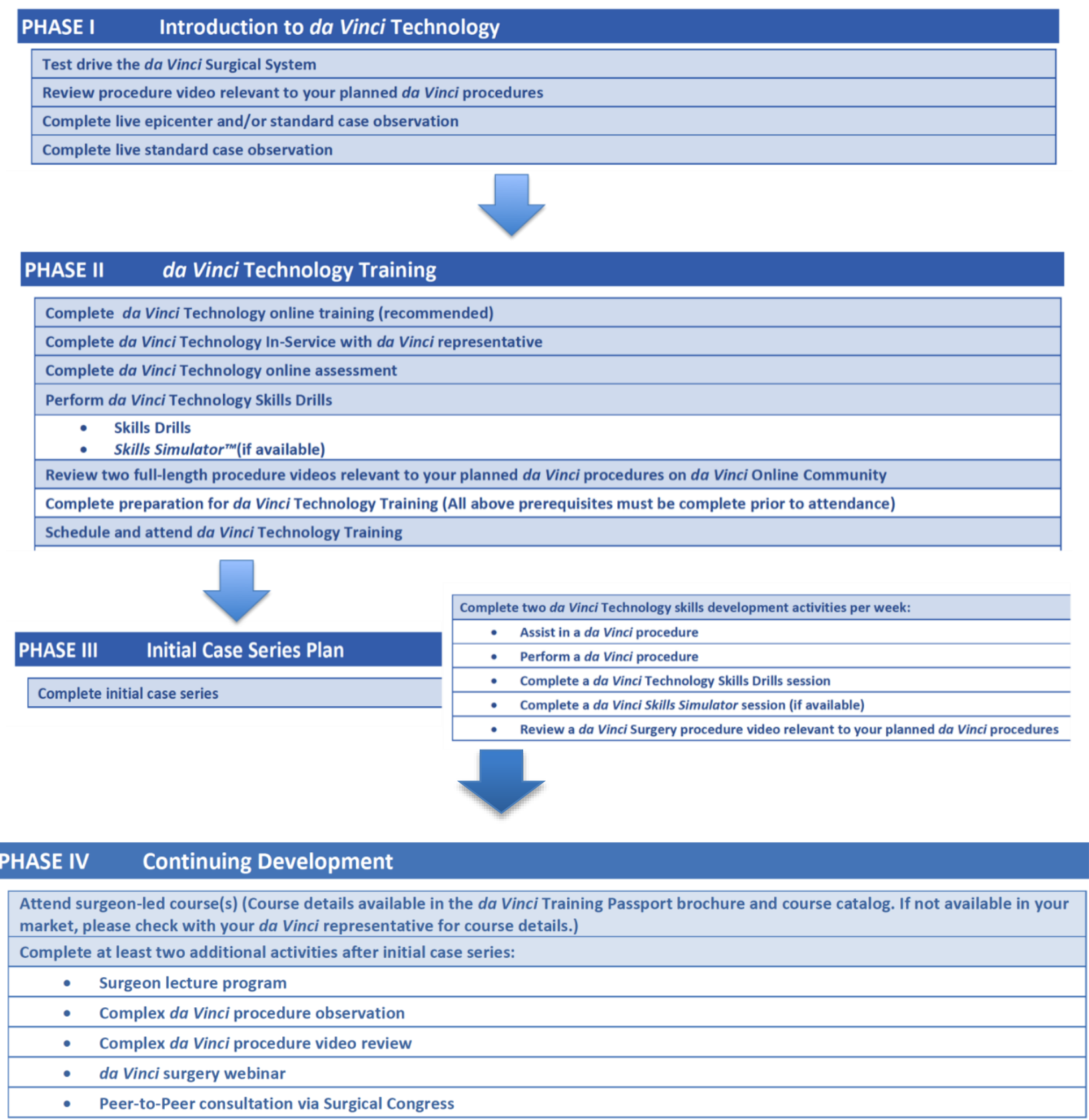

Figure 3 Da Vinci Phases to Certification 
In order to qualify for a Certificate of da Vinci System Training, must satisfy all of the phases listed below (Figs. 3).

- Introduction to da Vinci Technology

- Da Vinci Technology Training

- Initial Case Series Plan

- Continuing Development

In our experience new robotic surgeons who perform at least one case per week for the first 12-13 weeks typically advance through their learning curve more quickly and develop more confidence and familiarity with the use of the technology than those who perform their initial cases less frequently. In addition, we have observed, a proctor provides important guided training in the early robotic cases. One role of a proctor is to guide the surgeon and his/her surgery team through their initial cases to assist with their mastery of the technology [11].

\section{Training}

Training is the key to mastering any surgical technique and should be emphasized even more so when it comes to robotic surgery. One must learn new skills regarding hand-eye coordination and also become accustomed to the loss of touch sensation. To overcome these difficulties, we must take advantage of several tools, which are simulators, mentored cases, robots with dual consoles, and robotic courses. This makes it possible to bridge the gap between early surgical skills and effective surgical performance when using a robot in a clinical setting without subjecting patients to unnecessary risks [12].

\subsection{Simulation Training}

A simulator is an educational tool that allows interactive performance of a particular task in an environment that recreates or replicates a real-world clinical scenario. Surgical simulator training can be separated into two broad categories: physical (mechanical) simulators, wherein the task is performed under videoscopic guidance in the realworld environment and "virtual reality" (VR) simulators, wherein the task is performed on a computer-based platform using an artificially generated virtual environment [13].

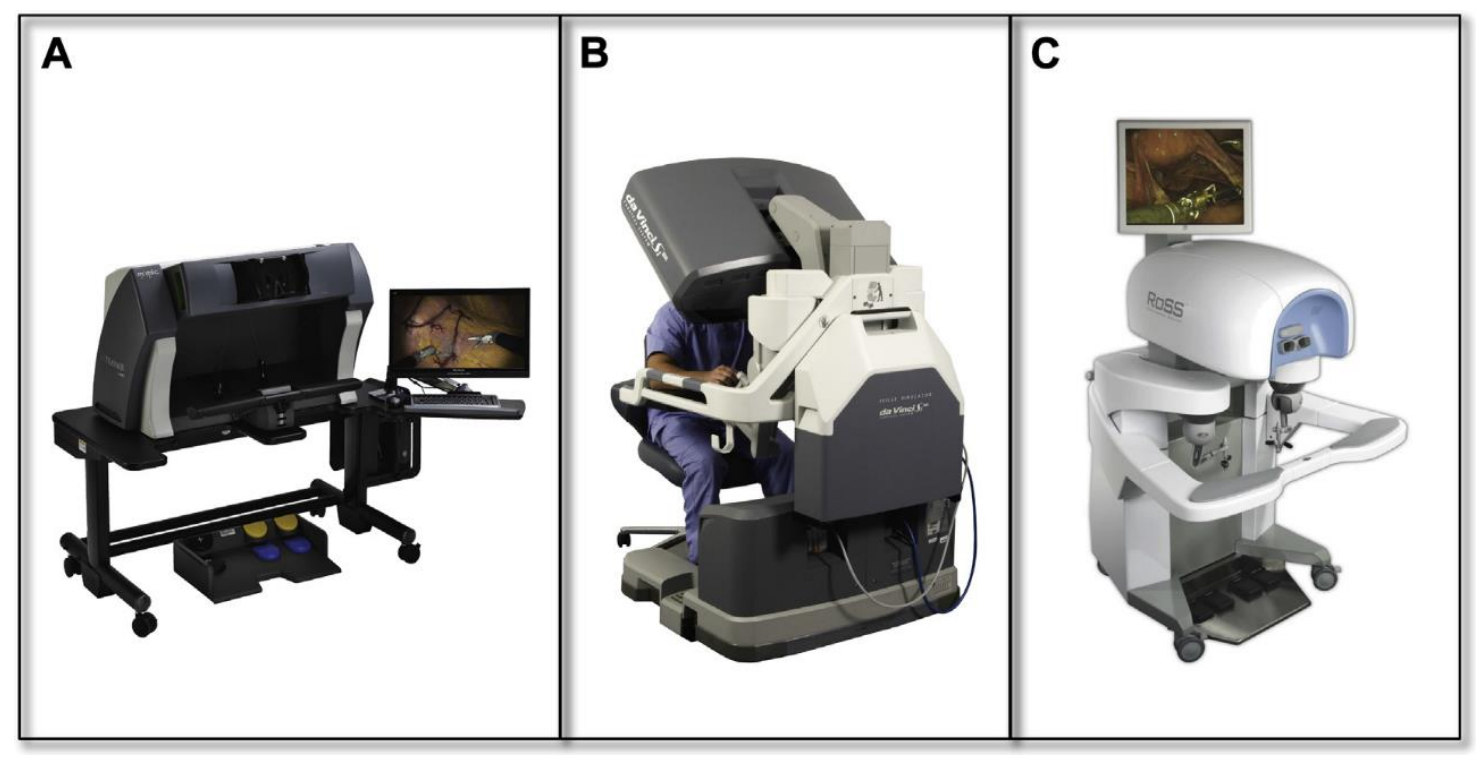

Figure 4 Three commercially available virtual reality simulator platforms. (A) dV Trainer; (B) da Vinci Skills Simulator; (C) Robotic Surgery Simulator.

There are five VR simulators currently available for robotic training. (Fig. 4) These include the Robotic Surgical Simulator (RoSS ${ }^{\mathrm{TM}}$; Simulated Surgical Systems, Buffalo, NY); dV-Trainer ${ }^{\mathrm{TM}}$ (Mimic Technologies, Inc., Seattle, WA); SEP Robot $^{\mathrm{TM}}$ (SimSurgery ${ }^{\mathrm{TM}}$, Norway); the da Vinci Skills Simulator (Intuitive Surgical, Sunnyvale, CA) and more recently the Robotix mentor $^{\mathrm{TM}}$ (3D systems, formerly Symbionix, Israel). All these simulators have been evaluated to have face validity (looks like what it simulates), content validity (accurately simulates the test condition) and construct validity [14-21]. 
The da Vinci Skills Simulator® dVSS (Fig. 5) consists of a small case that generates the virtual environment. The case is annexed to an existing da Vinci surgeon console, thereby transforming it into a simulation platform. The primary features are built-in metrics that enable the trainee to assess skills and to measure the improvement in a given exercise, with real-time feedback and progress tracking [16]. Exercises range from beginning to advanced and cover the following five categories: EndoWrist $®$ manipulation, camera and clutching, fourth arm integration, system setting, needle control and driving, as well as energy and dissection. During the developmental stages, several studies have shown face, construct, and content validity for the dVSS [17, 22-26]. Recently, Hung et al. [19] were able to demonstrate concurrent and predictive validity in a group of trainees who underwent a 10-week simulation course with dVSS by evaluating ex vivo tissue performance and final tissue performance after completion of the course. The dVSS uses the same console used for operative procedures, thus making it advantageous. However, the cost and logistics for securing a console of training purposes may be a prohibitive factor in some institutions [19].
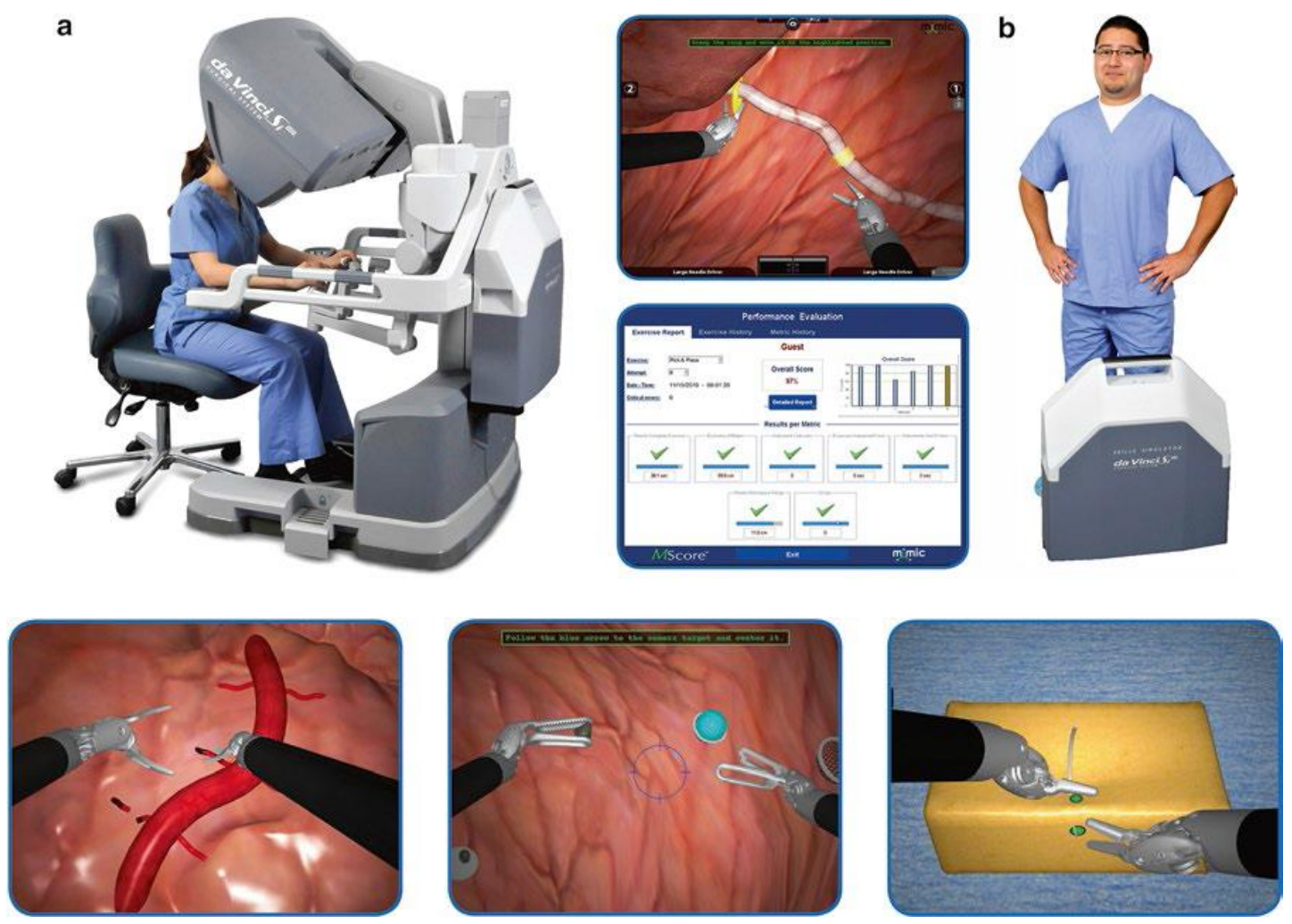

FI

Figure 5 da Vinci ${ }^{\circledR}$ surgeon console (a) and da Vinci® Skills Simulator (b) (Copyright (C) 2014 Intuitive Surgical, Inc. Used with permission)

\subsection{Dry Lab Training}

Dry lab simulation is cost effective and can reliably simulate cutting, suturing and grasping exercises. As the user is actually sitting on the daVinci ${ }^{\mathrm{TM}}$ surgical system (dVSS) console and using robotic instruments to complete tasks, the fidelity of simulations is very high. The consumables for dry lab exercises can be as simple as routine beads/needles/sutures, to sophisticated vascular and bowel models. Dry lab exercises are however limited in that it is difficult to maintain a standardized record or method of assessment, something that is essential in the early stage. Objective assessments made by a keen observing trainer are required in order for the trainee to have any benefit from the system [27-29].

\subsection{Wet Lab Training}

Handling of tissues and understanding the reaction of tissues to instrument touch cannot be learnt in dry labs. Further, use of diathermy and vascular control can only be learnt in wet labs. Experience in the wet lab soon teaches the trainee to recognize the consistency of tissues based solely on visual clues. Robotic wet labs provide excellent training ground 
for near live surgical exposure. Wet labs can provide three different types of training material. Frozen animal parts [30, 31], frozen human body parts [32] and live animals [33] with the cost increasing proportionately. Embalmed body parts allow vascular identification and dissection but alas do not provide a learning ground for vascular control. Live anesthetized or euthanized animal models are expensive and limited with regard to number of times they can be used, but have an advantage of bleeding simulation.

\subsection{Training in the Operating Room}

Once signed off on simulated skills, the trainee should be competent to learn the steps of the procedure on the console safely. This should follow a modular approach. The role of a mentor is crucial in this process. The trainee must enter into an agreement with the mentor who will oversee training. The modular process begins with the trainee performing the simplest part of the procedure, and progressively taking on increasingly difficult bits as the mentor sees fit. (Table 1) The transition of mentor from to proctor usually indicates that a trainee is progressing [34].

\subsection{Patient Side Training}

Like any surgical procedure, development of robotic skills follows a progression of observing, assisting, performing under supervision, and finally independent practice. Patient side training has a two-pronged benefit. It not only exposes the trainee to the steps of the operative procedure, but also necessitates the development of skills unique to the assistant. The assistant develops an understanding of the ergonomics and restriction of access created by the robotic arms. It is plausible that patient side skills are acquired relatively quickly, and that establishing a sign off of competency would enable progression to console in a relatively short duration [34].

Table 1 Relevant tasks for robotic surgery training and their rank order gathered by subject matter experts

\begin{tabular}{lll}
\hline Occurrence & Task name & Rank order \\
\hline Pre-op & & \\
\hline & Situation awareness & 1 \\
& Closed loop communication & 6 \\
Docking & 7 \\
Robotic Trocars & 8 \\
Ergonomic Positioning & 9 \\
System settings & 10 \\
Ontra-OR & Respond to robot system error & 11 \\
& Eye-hand instrument coordination & 2 \\
& Needle driving & 3 \\
& Atraumatic handling & 4 \\
Safety of operative field & 5 \\
\hline
\end{tabular}

\section{Patient Positioning and Port Placement}

Patient positioning and port placement play a key role in the ergonomics of the procedure. Proper patient positioning not only ensures that each member of the surgical team gets adequate access to the patient, but also maintains an optimal spatial configuration between the patient cart of the robot and the target organ in question. Similarly, correct port placement enables access to target organs, allowing for the required triangulation, without any extracorporeal or intracorporeal instrument clashes. [34]. 


\section{Case Observation}

It has been demonstrated that live case observation in an OR is an important component of the robotic training process. This phase of training affords the trainee the opportunity to watch more experienced surgeons perform live cases, thereby gaining an insight into the OR team dynamics, docking procedures, and troubleshooting of problems. The addition of a prerecorded video that can be watched with the teacher surgeon is another option. The video recording has the advantage that surgeries are selected in advance and the educational experiences can be preemptively planned [35].

\section{Proctoring}

Proctoring can take place during the initial phase of the learning curve as the surgeon begins to perform robotic cases. In proctoring, a master surgeon directly supervises and evaluates the skills and knowledge of a trainee. Proctoring can be an expensive form of training, but it provides a relatively safe way of introducing the new technique and serves as a transition phase to operating independently. In addition, the trainee can receive feedback and assessment from the proctor while performing the case. Proctoring can address important medico-legal aspects of training as well as have a role in institutional credentialing. [36].

\section{Dual Console}

Intuitive Surgical has developed a da Vinci ${ }^{\circledR}$ model that has an available dual console which will potentially allow for expert surgeon direction and supervision for procedural robotic training and collaboration. The mentoring console has two collaborative modes: (1) The swap mode allows the mentor and trainee to operate simultaneously and actively swap control of the robotic arms. (2) The nudge mode allows them to have control simultaneously, sharing the two robotic arms. Studies have shown that the swap mode was most useful during parts of the surgical procedures that required multiple hands (e.g., isolation and division of vessels). The nudge mode, however, was more useful for guiding the resident's hands during the more crucial and precise steps of an operation (e.g., suturing). The introduction of the dual console could shorten the learning curve and help trainees feel more comfortable when initially performing the procedure. This new robotic system could lead to safer educational training and also opens the gate to a whole new way of training, termed "telementoring," defined as the use of audiovisual technology at any distance to provide mentoring or teaching $[37,38]$.

\section{Robotic Courses}

Many guidelines have been published on how a robotic surgery course must be composed, the surgeon should learn about the robotic system components, draping the robot's arms, patient positioning, docking techniques, port placement strategy, inserting and exchanging instruments, and, importantly, basic system safety, emergency undocking procedure as well as dealing with troubleshooting errors and faults which may happen during the initial experience. This information should be provided first through lectures and then, after an examination of the surgeon's learned knowledge, transferred to the practical field through hands-on tutorials where the trainee can interact with the robot in a low-stress environment and apply what he has learned. This part of the course should allow a complete understanding of device function and technology altered functional status, and device parameters and limitations [3941].

\section{Credentialing Process}

The final stage in the robotic education process is obtaining credentialing privileges to operate independently. At present, there is no standardized credentialing process for robotic surgeons. Most requirements vary from hospital to hospital and are not competency based but rather driven by a fixed number of proctored cases. The issue of credentialing is attracting increasing attention, as there have been recent reports of litigation directed at hospitals resulting from insufficient training and credentialing of their robotic surgeons. For conventional laparoscopy, the Society of American Gastrointestinal and Endoscopic Surgeons (SAGES) have created the FLS curriculum, which has been validated as a means of training and credentialing trainees. Completion of this course has been endorsed by the American College of Surgeons as a prerequisite for board certification [39]. 


\section{Conclusion}

The use of robot-assisted laparoscopic surgery has increased rapidly, and with it, the need to better define a structured curriculum and credentialing process. Numerous efforts have been made by surgical societies to define the requisite skills for robotic surgeons, but in the United States, individual institutions have the responsibility for granting privileges.

In conclusion based on our early experience, we would recommend a road map of training with assessment of competency at every level before progression.

Such a training program would be based on demonstration of proficiency and safety in executing basic robotic skills and procedural tasks as well as achievement of non-technical skills in the practice laboratory prior to proceeding to modular training, and finally to sign off and independent practice. Recently, efforts have focused on creating a standardized curriculum with competency-based assessments. A competency-based approach offers a better hope of honoring the principle of "above all, do no harm" and obtaining continued acceptance of new operative technologies such as robotassisted surgery.

\section{Compliance with ethical standards}

\section{Acknowledgments}

None

\section{Disclosure of conflict of interest}

There are no conflicts of interest.

\section{References}

[1] Satava RM, Gallagher AG and Pellegrini CA. (2003). Surgical competence and surgical proficiency: definitions, taxonomy, and metrics. J Am Coll Surg, 196, 933-937.

[2] Allen D. (1907). The teaching of surgery. Trans Am Surg Assoc, 25, 1-14.

[3] Choussein S, Srouji SS, Farland LV, Wietsma A, Missmer SA, Hollis M, Yu RN, Pozner CN and Gargiulo AR. (2018). Robotic assistance confers ambidexterity to laparoscopic surgeons. J Minim Invasive Gynecol, 25, 76-83.

[4] Monn MF, Bahler CD, Schneider EB and Sundaram CP. (2013). Emerging trends in robotic pyeloplasty for the management of ureteropelvic junction obstruction in adults. J Urol, 189, 1352-1357.

[5] Yu HY, Hevelone ND, Lipsitz SR, Kowalczyk KJ and Hu JC. (2012). Use, costs and comparative effectiveness of robotic assisted, laparoscopic and open urological surgery. J Urol, 187, 1392-1398.

[6] Pinkerton S. (2013). The pros and cons of robotic surgery. The Wall Street Journal. November, 17.

[7] Clark CE, Turner JS, Kpodzo D, Reid KM, Hobson L, Moore C, Childs E, Clark K, Dansby M, Chase A and Johnson S. (2019). Adopting robotics training into a general surgery residency curriculum: where are we now? Curr Surg Rep, 7, 2.

[8] Intuitive Sunnyvale: da Vinci by intuitive.

[9] Peters BS, Armijo PR, Krause C, Choudhury SA and Oleynikov D. (2018). Review of emerging surgical robotic technology. Surg Endosc, 32, 1636-1655.

[10] Intuitive Sunnyvale: da Vinci by intuitive. English - da Vinci technology training pathway - Surgeon.

[11] Issenberg SB, McGaghie WC, Hart IR, Mayer JW, Felner JM, Petrusa ER, Waugh RA, Brown DD, Safford RR, Gessner IH, Gordon DL and Ewy GA. (1999). Simulation technology for health care professional skills training and assessment. JAMA, 282, 861-866.

[12] Abboudi H, Khan MS, Aboumarzouk O, Guru KA, Challacombe B, Dasgupta P and Ahmed K. (2013). Current status of validation for robotic surgery simulators - a systematic review. BJU Int, 111, 194-205. 
[13] Kesavadas T, Stegemann A, Sathyaseelan G, Chowriappa A, Srimathveeravalli G, Seixas-Mikelus S, Chandrasekhar R, Wilding G and Guru K. (2011). Validation of robotic surgery simulator (RoSS). Stud Health Technol Inform, 163, 274-276.

[14] Seixas-Mikelus SA, Kesavadas T, Srimathveeravalli G, Chandrasekhar R, Wilding GE and Guru KA. (2010). Face validation of a novel robotic surgical simulator. Urology, 76, 357-360.

[15] Kenney PA, Wszolek MF, Gould JJ, Libertino JA and Moinzadeh A. (2009). Face, content, and construct validity of dV-trainer, a novel virtual reality simulator for robotic surgery. Urology, 73, 1288-1292.

[16] Sethi AS, Peine WJ, Mohammadi Y and Sundaram CP. (2009). Validation of a novel virtual reality robotic simulator. J Endourol, 23, 503-508.

[17] Seixas-Mikelus SA, Stegemann AP, Kesavadas T, Srimathveeravalli G, Sathyaseelan G, Chandrasekhar R, Wilding GE, Peabody JO and Guru KA. (2011). Content validation of a novel robotic surgical simulator. BJU Int, 107, 11301135.

[18] Hung AJ, Patil MB, Zehnder P, Cai J, Ng CK, Aron M, Gill IS and Desai MM. (2012). Concurrent and predictive validation of a novel robotic surgery simulator: a prospective, randomized study. J Urol, 187, 630-637.

[19] Whittaker G, Aydin A, Raison N, Kum F, Challacombe B, Khan MS, Dasgupta P and Ahmed K. (2016). Validation of the robotix mentor robotic surgery simulator. J Endourol, 30, 338-346.

[20] Alzahrani T, Haddad R, Alkhayal A, Delisle J, Drudi L, Gotlieb W, Fraser S, Bergman S, Bladou F, Andonian S and Anidjar M. (2013). Validation of the da Vinci surgical skill simulator across three surgical disciplines: a pilot study. Can Urol Assoc J, 7, E520-E529.

[21] Lendvay TS, Casale P, Sweet R and Peters C. (2008). Initial validation of a virtual-reality robotic simulator. J Robot Surg, 2, 145-149.

[22] Lendvay TS, Casale P, Sweet R and Peters C. (2008). VR robotic surgery: randomized blinded study of the dVtrainer robotic simulator. Stud Health Technol Inform, 132, 242-244.

[23] Lerner MA, Ayalew M, Peine WJ and Sundaram CP. (2010). Does training on a virtual reality robotic simulator improve performance on the da Vinci surgical system? J Endourol, 24, 467-472.

[24] Kelly DC, Margules AC, Kundavaram CR, Narins H, Gomella LG, Trabulsi EJ and Lallas CD. (2012). Face, content, and construct validation of the da Vinci skills simulator. Urology, 79, 1068-1072.

[25] Hung AJ, Zehnder P, Patil MB, Cai J, Ng CK, Aron M, Gill IS and Desai MM. (2011). Face, content and construct validity of a novel robotic surgery simulator. J Urol, 186, 1019-1024.

[26] Ramos P, Montez J, Tripp A, Ng CK, Gill IS and Hung AJ. (2014). Face, content, construct and concurrent validity of dry laboratory exercises for robotic training using a global assessment tool. BJU Int, 113, 836-842.

[27] Siddiqui NY, Galloway ML, Geller EJ, Green IC, Hur HC, Langston K, Pitter MC, Tarr ME and Martino MA. (2014). Validity and reliability of the robotic objective structured assessment of technical skills. Obstet Gynecol, 123, 1193-1199.

[28] Siddiqui NY, Tarr ME, Geller EJ, Advincula AP, Galloway ML, Green IC, Hur HC, Pitter MC, Burke EE and Martino. (2016). Establishing benchmarks for minimum competence with dry lab robotic surgery drills. J Minim Invasive Gynecol, 23, 633-638.

[29] Polin MR, Siddiqui NY, Comstock BA, Hesham H, Brown C, Lendvay TS and Martino MA. (2016). Crowdsourcing: a valid alternative to expert evaluation of robotic surgery skills. Am J Obstet Gynecol, 215, 644.e641-e644.e647.

[30] Laguna MP, Arce-Alcazar A, Mochtar CA, Van Velthoven R, Peltier A and de la Rosette JJ. (2006). Construct validity of the chicken model in the simulation of laparoscopic radical prostatectomy suture. J Endourol, 20, 69-73.

[31] Huri E, Ezer M and Chan E. (2016). The novel laparoscopic training 3D model in urology with surgical anatomic remarks: fresh-frozen cadaveric tissue. Turk J Urol, 42, 224-229.

[32] Wagner A, Munter M, Makarov D, Nielsen M, Scorpio D and Kavoussi LR. (2008). Totally laparoscopic creation of a novel stapled orthotopic neobladder in the porcine model. J Endourol, 22, 151-156.

[33] Sridhar AN, Briggs TP, Kelly JD and Nathan S. (2017). Training in robotic surgery-an overview. Curr Urol Rep, 18, 58. 
[34] Nezhat C and Lakhi N. (2016). Learning experiences in robotic-assisted laparoscopic surgery. Best Pract Res Clin Obstet Gynaecol, 35, 20-29.

[35] Zorn KC, Gautam G, Shalhav AL, et al. (2009). Training, credentialing, proctoring and medicolegal risks of robotic urological surgery: recommendations of the society of urologic robotic surgeons. J Urol, 182, 1126-1132.

[36] Hanly EJ, Miller BE, Kumar R, Hasser CJ, Coste-Maniere E, Talamini MA, Aurora AA, Schenkman NS and Marohn MR. (2006). Mentoring console improves collaboration and teaching in surgical robotics. J Laparoendosc Adv Surg Tech A, 16, 445-451.

[37] Marengo F, Larrain D, Babilonti L and Spinillo A. (2012). Learning experience using the double-console da Vinci surgical system in gynecology: a prospective cohort study in a university hospital. Arch Gynecol Obstet, 285, 441445 .

[38] Rogula T, Acquafresca PA and Bazan M. (2015). Training and credentialing in robotic surgery. In: Kroh M, Chalikonda S (eds) Essentials of robotic surgery. Springer International Publishing, Cham, 13-26.

[39] Intuitive Surgical. (2014). Training pathway.

[40] Patel VR. (2006). Essential elements to the establishment and design of a successful robotic surgery programme. Int J Med Robot, 2, 28-35.

\section{How to cite this article}

Alejandro DGG, Regina FP, Fabiola NG and Eduardo VT. (2020). The road to becoming a certified robotic surgeon. World Journal of Advanced Research and Reviews, 7(1), 187-196. 